\title{
A portfolio selection using fuzzy analytic hierarchy process: A case study of Iranian pharmaceutical industry
}

\author{
Solmaz Ghazanfar Ahari ${ }^{a}$, Nader Ghaffari-Nasab ${ }^{b *}$, Ahmad Makui ${ }^{b}$, and Seyed Hassan Ghodsypour ${ }^{a}$ \\ ${ }^{a}$ Department of Industrial Engineering, Amirkabir University of Technology (Tehran Polytechnic), Tehran, Iran \\ ${ }^{b}$ Department of Industrial Engineering, Iran University of Science and Technology, Tehran, Iran

AR T I C LE I N F O \\ Article history: \\ Received 31 January 2010 \\ Received in revised form \\ 28 March 2010 \\ Accepted 1 May 2010 \\ Available online 7 May 2010 \\ Keywords: \\ Fuzzy AHP \\ Portfolio Selection

\begin{abstract}
Portfolio selection is one of the important problems encountered by any investor. The purpose of this paper is to solve a real stock portfolio selection problem in Iran. According to the uncertain environments in which financial decisions are made, most of the recent works in this pharmaceutical companies in Tehran stock exchange. For this purpose we apply two fuzzy analytic hierarchy process (FAHP) methods to this problem. Finally, the results obtained from the two methods are compared in terms of the solution quality.
\end{abstract} \\ field use fuzzy sets theory in order to incorporate these uncertainties into their analysis. The \\ problem is to determine how to allocate a limited fund among the stocks of some
}

Decision Making

Nonlinear Programming

Finance

\section{Introduction}

In multiple criteria decision making (MCDM) problems, a decision maker (DM) often needs to select or rank alternatives associated with some usually conflicting attributes or objectives. These problems arise in many real-world situations. For instance, in portfolio selection problems which arise frequently in financial management contexts, different financial attributes, such as profitability, mean rate of profit, and risk factors in a form of variance-covariance matrix, as well as some other related attributes are considered in the selection of a satisfactory portfolio. The asset allocation problem has been one of the most important subjects of research in the area of modern finance since the 1950s. In 1952, Markowitz proposed his pioneering work, the mean-variance method for the portfolio selection problem. This area of research has been under tremendous investigation using either quantitative or qualitative analysis. One of the most interesting and promising research topics in this area is the use of fuzzy set theory. Fuzzy set theory is a powerful tool used to describe an uncertain environment with ambiguity, vagueness or some other type of fuzziness, which appears in many aspects of financial markets, such as the unpredictable behavior of financial managers (Wang \& Zhu, 2002). In portfolio selection through the conventional AHP (Saaty et al., 1980), the basic approach is to set up a hierarchy consisting of the final goal of the problem or the decision to be made, a number of criteria, the sub-criteria associated with each main criterion, and a number of alternatives to select. In the

\footnotetext{
* Corresponding author. Tel./fax: +98-914-1044339.

E-mail addresses: Email address: nghaffari@ind.iust.ac.ir (N. Ghaffari-Nasab) 
problem of portfolio selection, the portfolio manager, as the decision maker, has a large set of criteria for selecting stocks. The problem is to compare the various criteria and to determine their relative importance through pairwise comparison between each pair of them. According to this rule, one can make a decision to compare each list of stocks with this criteria list and determine the amount of investment to be allocated to each stock. AHP is frequently used to solve the multiple criteria decision making problems and has successfully been applied for many practical contexts (Saaty, 1980). However, the method has been criticized because it cannot handle the inherent uncertainty and imprecision which are associated to mapping of DM's perceptions to exact numbers. Traditional AHP requires exact or crisp judgments (numbers). However, one may prefer to use fuzzy numbers for his/her judgment instead of using crisp numbers. Besides, even when people implement the same words, individual judgments of events may be different. Furthermore, even if the meaning of a word is thoroughly well-defined (e.g., the linguistic comparison labels in the standard AHP questionnaire responses), there are always some boundary criterion which are not well-defined and which make it difficult for the final judgment. One alternative to handle these difficulties is to use the concept of fuzzy numbers using linguistic variables associated with natural or artificial language. Linguistic variables are often used to represent the imprecise nature of human cognition when we try to translate people's opinions into spatial data. The preferences in AHP are necessarily human judgments which are from human perceptions, so fuzzy approaches allow for a more precise description of the decision-making process. There have been a number of methods developed to handle fuzzy AHP (Wang \& Zhu, 2002). In the literature, there are several approaches for constructing a portfolio. Historically, the mean-variance model is the first example of a portfolio optimization problem, and it is credited to Markowitz, who presented his ideas in 1952 (see Markowitz, 1991). However, by using AHP, Saaty (1980) analyzed more complex portfolio selection systems. Tanaka and Guo (1999) formulated portfolio selection models by quadratic programming, based on two kinds of possibility distributions. Xia et al. (2000) proposed a new model for portfolio selection using genetic algorithms. Inuiguchi and Ramik (2000) reviewed a number of fuzzy linear programming methods from a practical perspective and compared fuzzy mathematical programming approaches with those of stochastic programming. Tanaka et al. (2000), in another work, proposed two portfolio selection models based on fuzzy probabilities and possibility distributions, rather than conventional probability distributions as in Markowitz's model. Based on the worst regret to the portfolio selection, Inuiguchi and Tanino (2000) proposed a new possibilistic programming approach for portfolio optimization, considering how a model yields a distributive investment solution. Parra et al. (2001) formulated a fuzzy goal programming with fuzzy goals and fuzzy constraints, taking into account three criteria: return, risk and liquidity. Ong et al. (2005) proposed a method which incorporates the grey and possibilistic regression models in formulating a novel portfolio selection model. Lacagnina and Pecorella (2006) developed a multi-stage stochastic fuzzy program with soft constraints and recourse in order to capture both uncertainty and imprecision and used their program to solve a portfolio management problem. Huang et al. (2006) revised the conventional mean-variance method to determine the optimal portfolio selection under the conditions of the uncertainty. Terol et al. (2006) formulated a fuzzy compromise programming problem, using Sharpe's single- index model in a soft framework, in order to solve portfolio selection problems. Giove et al. (2006) considered a portfolio selection problem in which the prices of the securities are treated as interval variables. Zhang et al. (2007) proposed two kinds of portfolio selection models based on lower and upper possibilistic means and possibilistic variance and presented an algorithm which can derive the possibilistic efficient frontier of the problem. Tiryaki and Ahlatcioglu (2009) used the two constrained fuzzy AHP methods, developed by Enea and Piazza (2004), to the problem of portfolio selection in Istanbul stock exchange. They addressed some fallacies in the first model of Enea and Piazza and corrected it. They also showed that the second model of Enea and Piazza, which is used in this study, is superior to their first model in terms of uncertainty level of the solutions obtained. Branke et al. (2009) integrated an active set algorithm optimization for portfolio selection into a multi-objective evolutionary algorithm (MOEA). The idea behind their method is to let the MOEA come up with some convex subsets of the set of all feasible portfolios, solve a critical line algorithm for each subset, and then consolidate the 
partial solutions to form the solution of the original non-convex problem. Anagnostopoulos and Mamanis (2010) formulated the problem of portfolio selection as a three objective optimization problem in order to find tradeoffs between risk, return and the number of securities in the portfolio. Gao and Chu (2010) focus on the constrained portfolio selection problem, which is an extension to the standard Markowitz model, and developed an improved particle swarm optimization (IPSO) algorithm to solve it.

During the past two decades, there have been several approaches to fuzzy AHP proposed by various authors. van Laarhoven and Pedrycz (1983) are believed to the people who used this kind of method. In their implementation, elements in the reciprocal matrix were expressed by triangular fuzzy numbers. In contrast, Buckley (1985) used trapezoidal numbers to determine fuzzy comparison ratios. He criticized Laarhoven and Pedrycz's method because linear equations do not necessarily yield a unique result, and this method is only valid for triangular fuzzy numbers. Ruoning and Xiaoyan (1992) discussed the extensions of AHP to fuzzy environments and presented a procedure for constructing the fuzzy judgment matrix. Chang (1996) proposed a method which uses triangular fuzzy numbers for the pairwise comparison scale of fuzzy AHP and extent analysis for the synthetic extent values of pairwise comparisons. Mikhailov (2000) proposed a new Fuzzy Programming Method, based on a geometrical representation of the prioritization process. Enea and Piazza (2004) presented an extended implementation of AHP method where the constraints considered within fuzzy AHP in order to take into account all the available information. They also reported that more certain and reliable results can be achieved by considering all the information derived from the constraints. Kahraman (2008) presented a comprehensive literature survey of the most important fuzzy AHP methods and illustrated the performance of each method by means of solving a numerical example for each one. Seçme et al. (2009) proposed a fuzzy multi-criteria decision model to evaluate the performances of some banks. In their implementation, a fuzzy analytic hierarchy process (FAHP) and technique for order performance by similarity to ideal solution (TOPSIS) are integrated to evaluate the five largest commercial banks of Turkish banking sector in terms of several financial and nonfinancial indicators. Yang (2009) proposed a logarithm triangular fuzzy number-analytic hierarchy process (AHP) method expanding from the traditional AHP method to analyze the efficiency and advantages of supply chain in a scientific manner and validate the usability of methods on performance evaluation index system.

The rest of the paper is organized as follows. Sections 2 and 3 summarize the two fuzzy AHP methods originally presented by Enea and Piazza and van Laarhoven and Pedrycz, respectively. Section 4 explains the hierarchy constructed for the problem and discusses the details of the implementation of these methods for portfolio selection in Tehran stock exchange. The results obtained by the two methods are compared in terms of their quality characteristics of uncertainty level of the solutions. Finally, conclusion remarks are given in the last section to summarize the contribution of the paper.

\section{Constrained fuzzy AHP method}

One of the key questions on the implementation of any fuzzy programming is the choice of fuzzy numbers. This paper adopts a special type of fuzzy numbers called triangular fuzzy numbers. A triangular fuzzy number $\tilde{A}$ is fully characterized by the triple of real numbers $(l, m, u)$, where $l<m<$ $u$. The parameter $m$ gives the maximal grade of the membership function $\mu_{\tilde{A}}(x)$ (i.e., $\left.\mu_{\tilde{A}}(m)=1\right)$, and the parameters "l" and " $u$ " are the lower and the upper bounds of the field of the possible evaluations. Enea and Piazza (2004) applied the concept of "fuzzy arithmetic with requisite constraint" formulated by Klir (1997), to the fuzzy AHP in order to avoid situations in which the traditional mathematical operators give meaningless results when applied to fuzzy numbers. For example, let us consider two triangular fuzzy numbers $\tilde{A}=\left[A_{l}, A_{m}, A_{u}\right]$ and $\tilde{B}=\left[B_{l}, B_{m}, B_{u}\right]$ and the 
fuzzy arithmetic expression $\tilde{A} /(\tilde{A}+\tilde{B})$. The fuzzy arithmetic operation affected by an equality constraint is defined as follows,

$$
{ }^{\alpha}[\tilde{A} /(\tilde{A}+\tilde{B})]={ }^{\alpha}\left[A_{l} /\left(A_{l}+B_{u}\right) ; A_{u} /\left(A_{u}+B_{l}\right)\right] .
$$

As a numerical example, for the two triangular fuzzy numbers $\tilde{A}=[1,2,3]$ and $\tilde{B}=[2,3,4]$, without considering the equality constraint, the $\alpha$-cut with $\alpha=0$ of the operation $\tilde{A} /(\tilde{A}+\tilde{B})$ is equal to $\tilde{C}$ $=[1 /(3+4), 2 /(2+3), 3 /(1+2)]=[1 / 7,2 / 5,1]$. But, if the equality constraint is considered, the result is $\tilde{C}$ $=[1 /(1+4), 2 /(2+3), 3 /(3+2)]=[1 / 5,2 / 5,3 / 5]$. This result derives from the additional information employed. By using the data derived from the equality constraint, a smaller interval is achieved which does not have impossible values. As a result, a lower level of vagueness is achieved because a larger amount of information is used (see Enea and Piazza, 2004).

Let $S_{i}=\left(S_{l i}, S_{m i}, S_{u i}\right)$ be the fuzzy score for the element associated with the element in the $i$ th row of fuzzy pairwise comparison matrix, where the indices $l, m$ and $u$ denote its lower, medium and upper, respectively. According to constrained fuzzy AHP method proposed by Enea and Piazza (2004), $S_{m i}$ is calculated as follows,

$$
S_{m i}=\left(\prod_{j=1}^{n} m_{i j}\right)^{1 / n} / \sum_{k=1}^{n}\left[\left(\prod_{j=1}^{n} m_{k j}\right)^{1 / n}\right] \quad(i, j, k=1, . ., n)
$$

$S_{l i}$ can be evaluated using the crisp mathematical programming model:

$S_{l i}=\min \left[\left(\prod_{j=1}^{n} a_{i j}\right)^{1 / n}\right] / \sum_{k=1}^{n}\left[\left(\prod_{j=1}^{n} a_{k j}\right)^{1 / n}\right] \quad(i, j, k=1, . ., n)$

subject to

$$
a_{k j} \in\left[l_{k j}, u_{k j}\right], \quad \forall j>k ; a_{j k}=1 / a_{k j}, \forall j<k ; a_{j j}=1
$$

and similarly, $S_{u i}$ can be evaluated using the crisp mathematical programming model,

$$
S_{u i}=\max \left[\left(\prod_{j=1}^{n} a_{i j}\right)^{1 / n}\right] / \sum_{k=1}^{n}\left[\left(\prod_{j=1}^{n} a_{k j}\right)^{1 / n}\right] \quad(i, j, k=1, . ., n)
$$

subject to

$$
a_{k j} \in\left[l_{k j}, u_{k j}\right], \quad \forall j>k ; a_{j k}=1 / a_{k j}, \forall j<k ; a_{j j}=1
$$

\section{Van Laarhoven and Pedrycz's approach}

Van Laarhoven and Pedrycz (1983) offer an algorithm which is the direct extension of Saaty's AHP method. They identify the weights through the AHP operations and in their study, van Laarhoven and Pedrycz use the triangular fuzzy numbers and the computation steps are the same as those in crisp AHP. The Lootsma's logarithmic least-squares method is used to derive fuzzy weights and fuzzy performance scores. Van Laarhoven and Pedrycz's approach is shown by the following steps,

Step 1. Consult with the experts and obtain $n+1$ fuzzy reciprocal matrix that takes the following form as shown (4). 


$$
\widetilde{A}=\left[\begin{array}{cccc} 
& \widetilde{a}_{121} & & \widetilde{a}_{1 n 1} \\
& \widetilde{a}_{122} & \ldots & \widetilde{a}_{1 n 2} \\
(1,1,1) & \vdots & & \vdots \\
& \widetilde{a}_{12 P 12} & & \widetilde{a}_{1 n P 1 n} \\
\widetilde{a}_{211} & & & \widetilde{a}_{2 n 1} \\
\widetilde{a}_{212} & & & \widetilde{a}_{2 n 2} \\
\vdots & (1,1,1) & & \vdots \\
\widetilde{a}_{21 P 21} & & & \widetilde{a}_{2 n P 2 n} \\
\widetilde{a}_{n 11} & \widetilde{a}_{n 21} & & \\
\widetilde{a}_{n 12} & \widetilde{a}_{n 22} & \ldots & (1,1,1) \\
\vdots & \vdots & & \\
\widetilde{a}_{n 1 P n 1} & \widetilde{a}_{n 2 P n 2} & &
\end{array}\right]
$$

where $\tilde{a}_{i j k}$ is the fuzzy ratio representing the relative importance of $i$ th factor to $j$ th one from the $k$ th expert's point of view. Note that $p_{i j}$ is zero when no expert expresses his/her comparison ratios. It is also greater than one when more than one decision maker express their comparison ratios.

Step 2. Let $z_{i}=\left(l_{i}, m_{i}, u_{i}\right)$. Solve the following linear equations,

$$
\begin{aligned}
& l_{i}\left(\sum_{\substack{j=1 \\
j \neq i}}^{n} p_{i j}\right)-\sum_{\substack{j=1 \\
j \neq i}}^{n} p_{i j} u_{j}=\sum_{\substack{j=1 \\
j \neq i}}^{n} \sum_{k=1}^{p_{i j}}\left(\ln l_{i j k}\right), \quad \forall i \\
& m_{i}\left(\sum_{\substack{j=1 \\
j \neq i}}^{n} p_{i j}\right)-\sum_{\substack{j=1 \\
j \neq i}}^{n} p_{i j} m_{j}=\sum_{\substack{j=1 \\
j \neq i}}^{n} \sum_{k=1}^{p_{i j}}\left(\ln m_{i j k}\right), \quad \forall i \\
& u_{i}\left(\sum_{\substack{j=1 \\
j \neq i}}^{n} p_{i j}\right)-\sum_{\substack{j=1 \\
j \neq i}}^{n} p_{i j} l_{j}=\sum_{\substack{j=1 \\
j \neq i}}^{n} \sum_{\substack{i j \\
\text { jal }}}^{p_{j}}\left(\ln u_{i j k}\right), \quad \forall i
\end{aligned}
$$

As $\ln \left(l_{i j k}\right)$ and $\ln \left(l_{i j k}\right)$ are lower and upper values of $\ln \left(a_{i j k}\right)=-\ln \left(a_{j i k}\right)$, the following must hold true (see Eq. (5)),

$$
\ln \left(l_{i j k}\right)+\ln \left(l_{j i k}\right)=\ln \left(u_{i j k}\right)+\ln \left(u_{j i k}\right)=0 \quad \forall i, j, k .
$$

Eqs. (5) and (7) are linearly dependent, the same relationship holds for Eq. (6). Generally, the solution for Eqs. (5), (6), and (7) is given as:

$$
z_{i}=\left(l_{i}+t_{1}, m_{i}+t_{2}, u_{i}+t_{1}\right), \quad \forall i
$$

where $t_{1}$ and $t_{2}$ can be chosen arbitrarily.

Step 3. The right sides of the equations above are operated using logarithmic operations. Then we obtain the fuzzy weight in Eq. (10): 
$w_{i}=\left(\lambda_{1} \exp \left(l_{i}\right), \lambda_{2} \exp \left(m_{i}\right), \lambda_{3} \exp \left(u_{i}\right)\right)$

where

$$
\lambda_{1}=\left[\sum_{i=1}^{n} \exp \left(u_{i}\right)\right]^{-1} \quad \lambda_{2}=\left[\sum_{i=1}^{n} \exp \left(m_{i}\right)\right]^{-1} \quad \lambda_{3}=\left[\sum_{i=1}^{n} \exp \left(l_{i}\right)\right]^{-1}
$$

Equation (10) can also be used to determin $r_{i j}$, the performance score of the ith alternative under the jth criterion.

Step 4. Steps 1-3 are repeated several times until all reciprocal matrices are solved. With the fuzzy weights and performance scores, we can calculate the fuzzy utility for alternative $A_{i}$ as,

$u_{i}=\sum_{j=1}^{n} w_{j} r_{i j}$

\section{An application to Tehran stock exchange}

In this section, we explain the implementation of Enea and Piazza's method (see Enea and Piazza, 2004) for the portfolio selection in Tehran Stock Exchange (TSE).

\subsection{Constructing the hierarchy}

The constructed hierarchy consists of seven most important criteria which are: market share, sales to assets ratio, mean profit, liquidity, $\mathrm{P} / \mathrm{E}$, assets, and variance (risk). A comprehensive fundamental analysis have been performed on pharmaceutical sector in TSE and five companies of Alborz Co., Abidi Co., Sobhan Co., Jaber Co., and Sina Co. have been selected for the process of asset allocation. Fig. 1 gives an overall view of the fuzzy AHP hierarchy used in this paper.

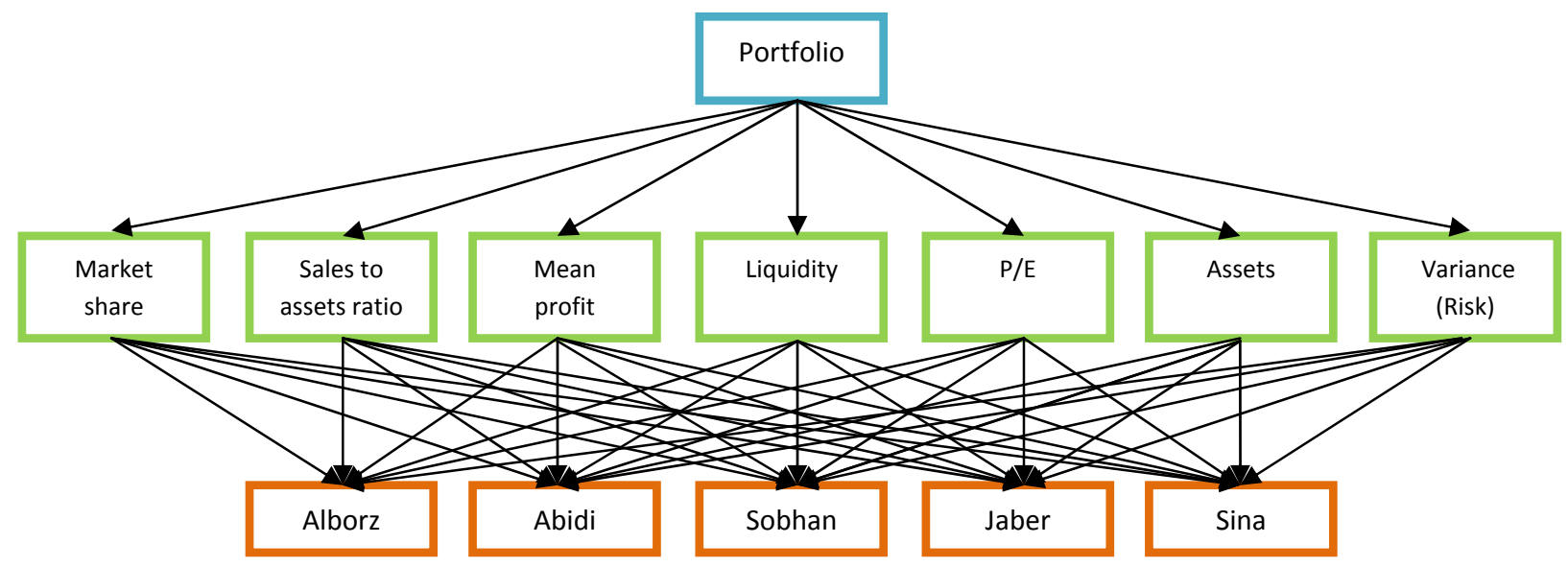

Fig. 1. Hierarchy of the problem

As we have already explained, we use linguistic variables to compare two evaluation criteria in fuzzy environment. For the "importance" criterion, we adopt the following five basic linguistic expressions to evaluate the degree of importance: "Just equal", "Equally important", "Weakly important", "Moderately important" and "Strongly important". The linguistic preferences or importance values expressed by the DM or expert are fuzzified using the triangular fuzzy numbers, which also fuzzify the linguistic preferences. Table 1 shows the triangular fuzzy conversion scale. The lower bound and the upper bound of triangular fuzzy numbers in Table 1 represent the uncertain range which might exist in the preferences expressed by the decision maker. The seven criteria are compared with 
respect to the goal "portfolio selection", and the corresponding fuzzy pairwise comparison matrix is solicited from the DM and presented in Table 2.

\section{Table 1}

Triangular fuzzy conversion scale

\begin{tabular}{lc}
\hline Linguistic importance value & Fuzzy pairwise comparison value \\
\hline Just equal & $(1,1,1)$, \\
Equally important & $(2 / 3,1,3 / 2)$ \\
Weakly important & $(3 / 2,2,5 / 2)$ \\
Moderately important & $(5 / 2,3,7 / 2)$ \\
Strongly important & $(7 / 2,4,9 / 2)$ \\
\hline
\end{tabular}

\section{Table 2}

Fuzzy pairwise comparison matrix for criteria with respect to goal "portfolio selection"

\begin{tabular}{cccccccc}
\hline Goal & C1 & C2 & C3 & C4 & C5 & C6 & C7 \\
\hline C1 & $(1,1,1)$ & $(7 / 2,4,9 / 2)$ & $(2 / 7,1 / 3,2 / 5)$ & $(2 / 3,1,3 / 2)$ & $(7 / 2,4,9 / 2)$ & $(7 / 2,4,9 / 2)$ & $(2 / 5,1 / 2,2 / 3)$ \\
C2 & $(2 / 9,1 / 4,2 / 7)$ & $(1,1,1)$ & $(2 / 9,1 / 4,2 / 7)$ & $(2 / 9,1 / 4,2 / 7)$ & $(2 / 9,1 / 4,2 / 7)$ & $(2 / 3,1,3 / 2)$ & $(2 / 9,1 / 4,2 / 7)$ \\
C3 & $(5 / 2,3,7 / 2)$ & $(7 / 2,4,9 / 2)$ & $(1,1,1)$ & $(3 / 2,2,5 / 2)$ & $(7 / 2,4,9 / 2)$ & $(7 / 2,4,9 / 2)$ & $(3 / 2,2,5 / 2)$ \\
C4 & $(2 / 3,1,3 / 2)$ & $(7 / 2,4,9 / 2)$ & $(2 / 5,1 / 2,2 / 3)$ & $(1,1,1)$ & $(7 / 2,4,9 / 2)$ & $(7 / 2,4,9 / 2)$ & $(2 / 5,1 / 2,2 / 3)$ \\
C5 & $(2 / 9,1 / 4,2 / 7)$ & $(7 / 2,4,9 / 2)$ & $(2 / 9,1 / 4,2 / 7)$ & $(2 / 9,1 / 4,2 / 7)$ & $(1,1,1)$ & $(7 / 2,4,9 / 2)$ & $(2 / 9,1 / 4,2 / 7)$ \\
C6 & $(2 / 9,1 / 4,2 / 7)$ & $(2 / 3,1,3 / 2)$ & $(2 / 9,1 / 4,2 / 7)$ & $(2 / 9,1 / 4,2 / 7)$ & $(2 / 9,1 / 4,2 / 7)$ & $(1,1,1)$ & $(2 / 9,1 / 4,2 / 7)$ \\
C7 & $(3 / 2,2,5 / 2)$ & $(7 / 2,4,9 / 2)$ & $(2 / 5,1 / 2,2 / 3)$ & $(3 / 2,2,5 / 2)$ & $(7 / 2,4,9 / 2)$ & $(7 / 2,4,9 / 2)$ & $(1,1,1)$ \\
\hline
\end{tabular}

The five mentioned stocks are now compared with respect to all the criteria in the hierarchy. The corresponding seven fuzzy pairwise comparison matrices are solicited from the DM. For the sake of simplicity we only report only one of them which is the fuzzy pairwise comparison matrix of market share, $\mathrm{C} 1$, in Table 3. This would help us understand the details of the mathematical programming problem used for this empirical study which is based on the method developed by Enea and Piazza.

\subsection{Applying the constrained fuzzy AHP method to Tehran stock exchange}

To solve our main problem of stock selection, the mathematical programming models (2) and (3) and formula (1) are implemented to calculate the final scores for each stock in the hierarchy. Let us use the fuzzy pairwise comparison matrix for the stocks with respect to market share, $C 1$, given in Table 3 .

\section{Table 3}

Fuzzy pairwise comparison matrix for alternatives with respect to $\mathrm{C} 1$

\begin{tabular}{cccccc}
\hline C1 & A1 & A2 & A3 & A4 & A5 \\
\hline A1 & $(1,1,1)$ & $(3 / 2,2,5 / 2)$ & $(2 / 3,1,3 / 2)$ & $(2 / 5,1 / 2,2 / 3)$ & $(2 / 3,1,3 / 2)$ \\
A2 & $(2 / 5,1 / 2,2 / 3)$ & $(1,1,1)$ & $(2 / 5,1 / 2,2 / 3)$ & $(2 / 5,1 / 2,2 / 3)$ & $(2 / 3,1,3 / 2)$ \\
A3 & $(2 / 3,1,3 / 2)$ & $(3 / 2,2,5 / 2)$ & $(1,1,1)$ & $(2 / 3,1,3 / 2)$ & $(2 / 3,1,3 / 2)$ \\
A4 & $(3 / 2,2,5 / 2)$ & $(3 / 2,2,5 / 2)$ & $(2 / 3,1,3 / 2)$ & $(1,1,1)$ & $(2 / 3,1,3 / 2)$ \\
A5 & $(2 / 3,1,3 / 2)$ & $(2 / 3,1,3 / 2)$ & $(2 / 3,1,3 / 2)$ & $(2 / 3,1,3 / 2)$ & $(1,1,1)$ \\
\hline
\end{tabular}

To calculate the $S_{l 1}$ for the first alternative A1: Alborz Co., according to (2), the following nonlinear mathematical programming problem is solved:

$$
\begin{aligned}
S_{l 1}= & \min \left\{\left(x_{1} x_{2} x_{3} x_{4}\right)^{1 / 5} /\left[\left(x_{1} x_{2} x_{3} x_{4}\right)^{1 / 5}+\left(x_{5} x_{6} x_{7} / x_{1}\right)^{1 / 5}+\left(x_{8} x_{9} / x_{2} x_{5}\right)^{1 / 5}+\left(x_{10} / x_{3} x_{6} x_{8}\right)^{1 / 5}+\left(1 / x_{4} x_{7} x_{9} x_{10}\right)^{1 / 5}\right]\right\} \\
\text { s.t }: \quad & 3 / 2 \leq x_{1} \leq 5 / 2 ; 2 / 3 \leq x_{2} \leq 3 / 2 ; 2 / 5 \leq x_{3} \leq 2 / 3 ; 2 / 3 \leq x_{4} \leq 3 / 2 ; 2 / 5 \leq x_{5} \leq 2 / 3 \\
& 2 / 5 \leq x_{6} \leq 2 / 3 ; 2 / 3 \leq x_{7} \leq 3 / 2 ; 2 / 3 \leq x_{8} \leq 3 / 2 ; 2 / 3 \leq x_{9} \leq 3 / 2 ; 2 / 3 \leq x_{10} \leq 3 / 2
\end{aligned}
$$


The resulted problem formulation is a special form of the geometric programming called posynomial where one can find the global optimum of the resulted problem using the recent advances of nonlinear programming summarized in a software package called CVX (Grant and Boyd, 2009) which yields the following optimal solution,

$S_{l 1}=0.143 ; x_{1}=1.500 ; x_{2}=0.667 ; \quad x_{3}=0.400 ; \quad x_{4}=0.667 ; \quad x_{5}=0.400 ; \quad x_{6}=0.400 ; \quad x_{7}=0.667 ; \quad x_{8}=0.667 ;$ $x_{9}=1.500 ; x_{10}=1.500$.

The implementation of the constrained fuzzy AHP method gives us the fuzzy weights for each criterion as in Table 4. The fuzzy scores for each criterion are summarized in Table 5 for each stock.

\section{Table 4}

Fuzzy weight of criteria (constrained fuzzy AHP method)

\begin{tabular}{lc}
\hline Criteria & Fuzzy triangular number \\
\hline C1: Market share & $(0.128,0.158,0.195)$ \\
C2: Sales to assets ratio & $(0.035,0.042,0.051)$ \\
C3: Mean profit & $(0.244,0.291,0.334)$ \\
C4: Turnover rate & $(0.134,0.167,0.209)$ \\
C5: P/E & $(0.065,0.076,0.089)$ \\
C6: Assets & $(0.035,0.042,0.051)$ \\
C7: Variance (risk) & $(0.185,0.225,0.268)$ \\
\hline
\end{tabular}

\section{Table 5}

Fuzzy score of stocks under each criterion (constrained fuzzy AHP method)

\begin{tabular}{cccccc}
\hline & A1 & A2 & A3 & A & A5 \\
\hline C1 & $(0.143,0.193,0.257)$ & $(0.099,0.135 .0 .166)$ & $(0.160,0.222,0.298)$ & $(0.191,0.255,0.326)$ & $(0.135,0.193,0.271)$ \\
C2 & $(0.324,0.381,0.431)$ & $(0.171,0.219,0.277)$ & $(0.047,0.055,0.064)$ & $(0.171,0.219,0.277)$ & $(0.104,0.126,0.155)$ \\
C3 & $(0.244,0.288,0.338)$ & $(0.326,0.380,0.429)$ & $(0.100,0.120,0.146)$ & $(0.046,0.053,0.062)$ & $(0.131,0.159,0.190)$ \\
C4 & $(0.126,0.151,0.180)$ & $(0.126,0.151,0.180)$ & $(0.446,0.486,0.518)$ & $(0.116,0.151,0.195)$ & $(0.053,0.063,0.075)$ \\
C5 & $(0.137,0.173,0.217)$ & $(0.168,0.229,0.299)$ & $(0.151,0.199,0.253)$ & $(0.140,0.199,0.273)$ & $(0.140,0.199,0.273)$ \\
C6 & $(0.101,0.131,0.192)$ & $(0.086,0.108,0.154)$ & $(0.151,0.248,0.255)$ & $(0.378,0.406,0.476)$ & $(0.086,0.108,0.154)$ \\
C7 & $(0.097,0.125,0.167)$ & $(0.202,0.250,0.299)$ & $(0.097,0.125,0.167)$ & $(0.202,0.250,0.299)$ & $(0.185,0.250,0.321)$ \\
\hline
\end{tabular}

Therefore, we can calculate the final fuzzy scores for each stock by multiplying the fuzzy scores by the associated fuzzy weight. The results are shown in Table 6 and Figure 2.

\section{Table 6}

Fuzzy final scores for stocks (constrained fuzzy AHP method)

\begin{tabular}{ccccc}
\hline A1 & A2 & A3 & A4 & A5 \\
\hline$(0.136,0.202,0.296)$ & $(0.166,0.244,0.342)$ & $(0.139,0.207,0.299)$ & $(0.117,0.178,0.268)$ & $(0.106,0.168,0.258)$ \\
\hline
\end{tabular}

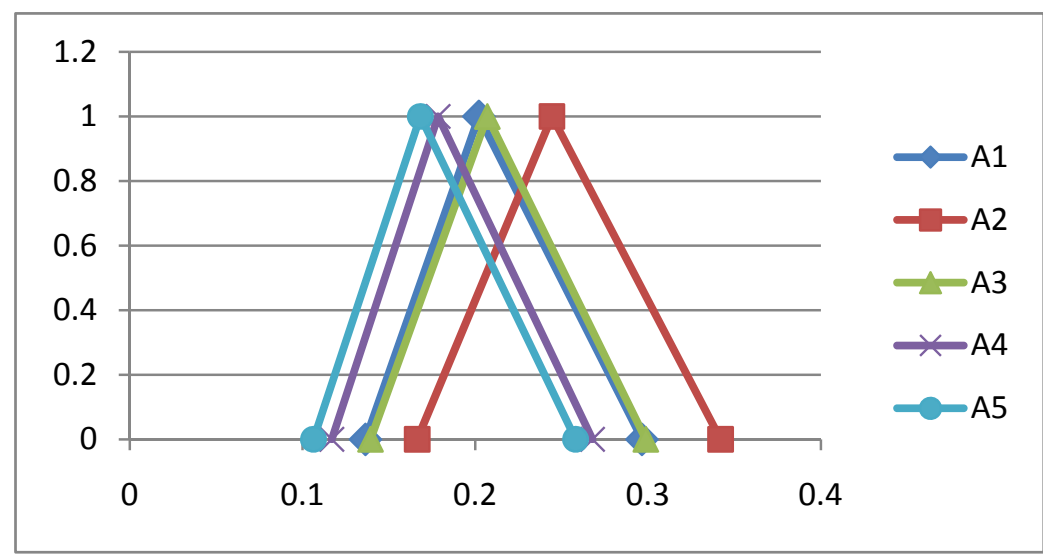

Fig. 2. Final fuzzy scores of alternatives (constrained fuzzy AHP method) 


\subsection{Applying van Laarhoven and Pedrycz's fuzzy AHP method to Tehran stock exchange}

In this section, we apply the fuzzy AHP method proposed by van Laarhoven and Pedrycz to get the weights for each criterion and the results are summarized in Table 7. Table 8 also summarizes the details of the information for the fuzzy numbers under different criteria.

\section{Table 7}

Fuzzy weight of criteria (van Laarhoven and Pedrycz's method)

\begin{tabular}{lc}
\hline Criteria & Weight \\
\hline C1: Market share & $(0.128,0.097,0.197)$ \\
C2: Sales to assets ratio & $(0.035,0.042,0.050)$ \\
C3: Mean profit & $(0.239,0.295,0.345)$ \\
C4: Turnover rate & $(0.134,0.169,0.213)$ \\
C5: P/E & $(0.067,0.126,0.086)$ \\
C6: Assets & $(0.035,0.042,0.050)$ \\
C7: Variance (risk) & $(0.183,0.228,0.274)$ \\
\hline
\end{tabular}

\section{Table 8}

Fuzzy score of stocks under each criterion (van Laarhoven and Pedrycz's method)

\begin{tabular}{lccccc}
\hline & A1 & A2 & A3 & A4 & A5 \\
\hline C1 & $(0.140,0.194,0.305)$ & $(0.099,0.129,0.195)$ & $(0.152,0.224,0.365)$ & $(0.183,0.257,0.396)$ & $(0.052,0.195,0.336)$ \\
C2 & $(0.308,0.381,0.461)$ & $(0.163,0.219,0.295)$ & $(0.049,0.055,0.062)$ & $(0.163,0.219,0.294)$ & $(0.105,0.126,0.156)$ \\
C3 & $(0.238,0.288,0.351)$ & $(0.311,0.381,0.457)$ & $(0.100,0.120,0.147)$ & $(0.048,0.053,0.061)$ & $(0.130,0.158,0.192)$ \\
C4 & $(0.130,0.151,0.175)$ & $(0.130,0.151,0.175)$ & $(0.443,0.485,0.525)$ & $(0.113,0.151,0.201)$ & $(0.056,0.063,0.072)$ \\
C5 & $(0.172,0.173,0.211)$ & $(0.163,0.229,0.262)$ & $(0.146,0.199,0.269)$ & $(0.123,0.199,0.308)$ & $(0.128,0.199,0.308)$ \\
C6 & $(0.101,0.139,0.193)$ & $(0.089,0.114,0.150)$ & $(0.154,0.199,0.255)$ & $(0.391,0.432,0.466)$ & $(0.089,0.114,0.150)$ \\
C7 & $(0.095,0.125,0.171)$ & $(0.201,0.250,0.305)$ & $(0.095,0.125,0.171)$ & $(0.201,0.250,0.305)$ & $(0.176,0.250,0.349)$ \\
\hline
\end{tabular}

Finally, Table 9 and Figure 3 summarize the ranking of all five alternatives for the implementation of the second proposed method.

\section{Table 9}

Fuzzy final scores for stocks (van Laarhoven and Pedrycz's method)

$\begin{array}{lllll}\text { A1 } & \text { A2 } & \text { A3 } & \text { A4 } & \text { A5 }\end{array}$

\begin{tabular}{llllll}
\hline $0.136,0.202,0.316)$ & $(0.161,0.250,0.362)$ & $(0.137,0.204,0.320)$ & $(0.115,0.176,0.290)$ & $(0.093,0.168,0.285)$
\end{tabular}

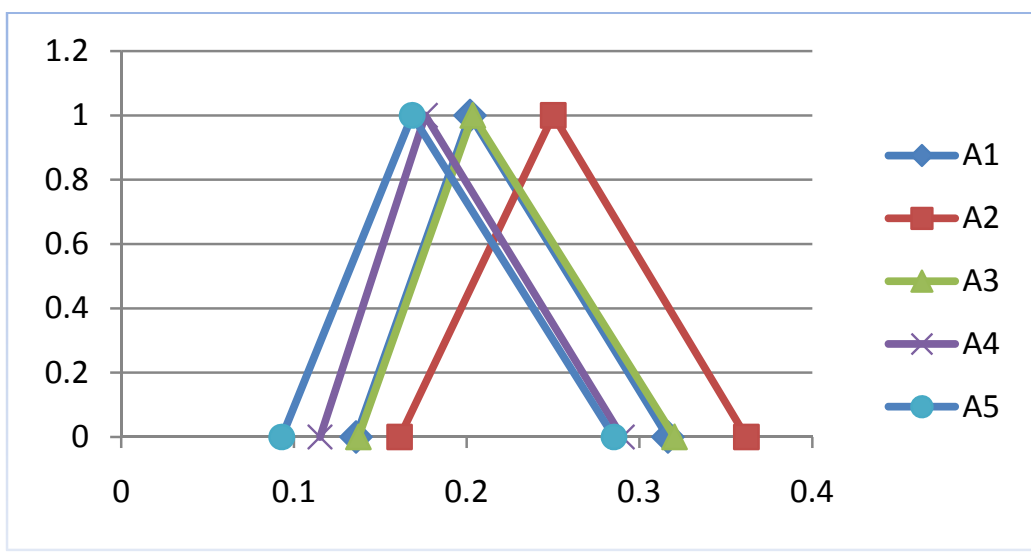

Fig. 3. Final fuzzy scores for five alternatives (van Laarhoven and Pedrycz's method) 
From Tables 6 and 9, and Figures 2 and 3 we can conclude that there is a small difference between the fuzzy numbers describing the final scores which are obtained by the two methods. In fact the scores obtained using constrained fuzzy AHP method contains a minor uncertainty level, as their $\alpha$ cuts are smaller. This is due to the fact that this method takes care of the constraints. In order to study the effects of uncertainty on the implementation of the two methods, we use a measure of Uuncertainty. When the fuzzy set is defined on $\square$, as it is normal, the $\alpha$-cuts ${ }^{\alpha} A$ are infinite sets (e.g., intervals of real number), ${ }^{\alpha} A$ is measurable and Lebesgue-integrable function $\mu\left({ }^{\alpha} A\right)$ is the measure of ${ }^{\alpha} A$ and $U(A)$ can be calculated as follows,

$$
U(A)=\int_{0}^{1} \log \left[1+\mu\left({ }^{\alpha} A\right)\right] \mathrm{d} \alpha
$$

If $\mathrm{A}=(l, m, u)$ is a triangular fuzzy number then the $U$-uncertainty is,

$$
\begin{aligned}
& U(A)=\left[-\frac{1}{u-1}[(1+u-l)-\alpha(u-1)] \cdot \ln [(1+u-l)-\alpha(u-1)]-\alpha\right]_{0}^{1} \\
& U(A)=-1+\frac{(1+u-l)}{(u-1)} \ln (1+u-l)
\end{aligned}
$$

Using the formula (13) on the results obtained with two different methods, one can easily obtain a measure of the $U$-uncertainty of the results which are showed in Table 10.

\section{Evaluation of the weights}

To evaluate a crisp weight for each stock, one can use the defuzzification method to replace the fuzzy numbers by crisp numbers. A ranking method which uses the defuzzification function is as follows:

$$
F(A)=\frac{1}{2} \int_{0}^{1}\left[{ }^{\alpha} \underline{a}+{ }^{\alpha} \bar{a}\right] \mathrm{d} \alpha
$$

where $\underline{a}$ and $\bar{a}$ are the infimum and supremum of the $\alpha$-cut of the fuzzy number $A$ defined for $x \in R$ , respectively.

\section{Table 10}

U-uncertainty measure of the results.

\begin{tabular}{lcc}
\hline & Constrained fuzzy AHP method & van Laarhoven and Pedrycz's method \\
\hline A1 & 0.0761 & 0.0853 \\
A2 & 0.0831 & 0.0943 \\
A3 & 0.0757 & 0.0864 \\
A4 & 0.0719 & 0.0828 \\
A5 & 0.0723 & 0.0905 \\
\hline
\end{tabular}

It is easy to show that the right hand side of the formula above equals: $F(A)=(l+2 m+u) / 4$ for triangular fuzzy numbers. Applying formula (14) to the overall fuzzy final scores derived from constrained fuzzy AHP method and van Laarhoven and Pedrycz's fuzzy AHP method, we obtain an evaluation of the precise weight of each stock as in Tables 11 and 12, respectively.

Table 11

Evaluation of the precise weight of stocks ( constrained fuzzy AHP method)

\begin{tabular}{ccccc}
\hline A1 & A2 & A3 & A4 & A5 \\
\hline 0.203 & 0.241 & 0.206 & 0.180 & 0.170 \\
\hline
\end{tabular}

Table 12

Evaluation of the precise weight of stocks (van Laarhoven and Pedryc's method)

\begin{tabular}{ccccc}
\hline A1 & A2 & A3 & A4 & A5 \\
\hline 0.203 & 0.242 & 0.205 & 0.180 & 0.170 \\
\hline
\end{tabular}


As we can observe from Table 11, using the constrained fuzzy AHP method, the solution states that the investor could allocate his/her fund in ratios of $20.3 \%, 24.1 \%, 20.6 \%, 18.0 \%, 17.0 \%$ to the alternatives A1 through A5, respectively. Also according to Table 12, the investor could allocate his/her fund in ratios of $20.3 \%, 24.2 \%, 20.5 \%, 18.0 \%, 17.0 \%$ to the alternatives A1 through A5, respectively.

\section{Conclusion}

In this paper, we have presented an empirical analysis of two fuzzy methods for a real world case study of pharmaceutical industry where all input parameters are subject to uncertainty. The results of our implementation determine the asset allocation for the common shares of five different companies when historical data is either unavailable or unreliable. The study uses the data from the shares of publicly traded companies in Tehran stock exchange. Our study first has determined the hierarchy of different parameters using eliciting related linguistic data from experts in the related area. Then, we have implemented the two fuzzy AHP methods: constrained FAHP method proposed by Enea and Piazza (2004) and van Laarhoven and Pedrycz's FAHP method (1983). The results of our implementation have been discussed and both methods have been compared.

\section{Acknowledgments}

We would like to express our appreciation to the personnel of the Tehran stock exchange for their sincere cooperation and guidance during the data gathering phase of this project. We also would like to thank the anonymous referee for his/her constructive comments on the earlier version of this work.

\section{References}

Anagnostopoulos, K.P., \& Mamanis, G. (2010). A portfolio optimization model with three objectives and discrete variables, Computers and Operations Research, 37(7), 1285-1297.

Buckley, J.J. (1985). Fuzzy hierarchical analysis, Fuzzy Sets and Systems, 17, 233-247.

Branke, J., Scheckenbach, B., Stein, M., Deb, K., \& Schmeck, H. (2009). Portfolio optimization with an envelope-based multi-objective evolutionary algorithm, European Journal of Operational Research, 199 (3), 684-693.

Chang, D.-Y. (1996). Applications of the Extent Analysis Method on Fuzzy AHP, European Journal of Operational Research, 95, 649-655.

Enea, M., \& Piazza, T. (2004). Project selection by constrained fuzzy AHP, Fuzzy Optimization and Decision Making, 3, 39-62.

Fortemps, F., \& Roubens, M. (1996). Ranking and Defuzzification Methods Based on Area Compensation, Fuzzy Sets and Systems, 82, 319-330.

Gao, J., \& Chu, Z. (2010). A new particle swarm optimisation based on MATLAB for portfolio selection problem, International Journal of Modelling, Identification and Control, 9(1-2), 206211.

Giove, S., Funari, S., \& Nardelli, C. (2006). An interval portfolio selection problem based on regret function, European Journal of Operational Research, 170(1), 253-264.

Grant, M., Boyd, S. (2009). www.stanford.edu/ boyd/cvxbook/.

Huang, J.-J., Tzeng, G.-H., \& Ong, C.-S., (2006). A novel algorithm for uncertain portfolio selection, Applied Mathematics and Computation, 173(1), 350-359.

Lacagnina, V., \& Pecorella, A. (2006). A stochastic soft constraints fuzzy model for a portfolio selection problem, Fuzzy Sets and Systems, 157(10), 1317-1327.

Inuiguchi, M \& Ramik, J. (2000). Possibilistic linear programming: a brief review of fuzzy mathematical programming and a comparison with stochastic programming in portfolio selection problem, Fuzzy Sets and Systems, 111(1), 3-28. 
Inuiguchi, M., \& T. Tanino, T. (2000). Portfolio selection under independent possibilistic information, Fuzzy Sets and Systems, 115(1), 83-92.

Kahraman, C. (Ed.). (2008). Fuzzy multi-criteria decision making, Springer, New York.

Klir, G. J. (1997). Fuzzy Arithmetic with Requisite Constraints, Fuzzy Sets and Systems, 91, 165175.

Markowitz, H. M. (1991). Foundations of portfolio theory, The Journal of Finance, 46(2), 469-477.

Mikhailov, L. (2000). A fuzzy programming method for deriving priorities in the analytic hierarchy process, Journal of Operational Research Society, 51, 341-349.

Ong, C.-S., Huang, J. J., \& Tzeng, G.-T. (2005). A novel hybrid model for portfolio selection, Applied Mathematics and Computation, 169(2), 1195-1210.

Parra, M. A., Terol, A. B., \& Uria, M. V. R. (2001). A fuzzy goal programming approach to portfolio selection, European Journal of Operational Research, 113(2), 287-297.

Ruoning, X., \& Xiaoyan, Z. (1992). Extension of the Analytic Hierarchy Process in Fuzzy Environment, Fuzzy Sets and Systems, 52, 251-257.

Saaty, T.L. (1980). The Analytic Hierarchy Process, McGraw-Hill, New York.

Saaty, T.L., Rogers, P.C., \& Bell, R. (1980). Portfolio selection through hierarchies, The Journal of Portfolio Management, 16-21.

Seçme, N.Y., Bayrakdaro lu, \& A., Kahraman, C. (2009). Fuzzy performance evaluation in Turkish Banking Sector using Analytic Hierarchy Process and TOPSIS, Expert Systems with Applications, 36(9), 11699-11709.

Tanaka, H., \& Guo, P. (1999). Portfolio selection based on upper and lower exponential possibility distributions, European Journal of Operational Research, 114, 115-126.

Tanaka, H., Guo, P., \& Türksen, I. B. (2000). Portfolio selection based on fuzzy probabilities and possibility distributions, Fuzzy Sets and Systems, 111(3), 387-397.

Terol, A. B., Gladish, B. P., Parra, M. A., \& Uria, M. V. R. (2006). Fuzzy compromise programming for portfolio selection, Applied Mathematics and Computation, 173 (1), 251-264.

Tiryaki, F., \& Ahlatcioğlu, B. (2009). Fuzzy portfolio selection using fuzzy analytic hierarchy process, Information Science, 179, 53-69.

Van Laarhoven, P. J. M., \& Pedrycz, W. (1983). A Fuzzy Extension of Saaty's Priority Theory, Fuzzy Sets and Systems, 11, 229-241.

Wang, S., \& Zhu, S. (2002). On fuzzy portfolio selection problems, Fuzzy Optimization and Decision Making, 1, 361-377.

Xia, Y., Liu, B., Wang, S., \& Lai, K.K. (2000). A model for portfolio selection with order of expected returns, Computers and Operations Research, 27 (5), 409-422.

Yang, J. (2009). Integrative performance evaluation for supply chain system based on logarithm triangular fuzzy number-AHP method, Kybernetes, 38(10), 1760-1770.

Zhang, W.-G., Wang, Y.-L., Chen, Z.-P., \& Nie, Z.-K. (2007). Possibilistic mean-variance models and efficient frontiers for portfolio selection problem, Information Sciences, 177(13), 27872801 . 